\title{
E-LEARNING: UN ANÁLISIS DESDE EL PUNTO DE VISTA DEL ALUMNO
}

\author{
(E-LEARNING: AN ANALYSIS FROM THE STUDENTS' POINT OF VIEW)
}

Eva Martínez Caro
Universidad Politécnica de Cartagena (España)

\section{RESUMEN}

El e-learning ofrece numerosas ventajas a organizaciones, universidades y usuarios, su mercado crece continuamente y son considerables las iniciativas existentes para su impulso. Sin embargo, también se han detectado algunos problemas que dificultan su implantación. Son muchos los estudios que tratan sobre esos aspectos pero en muchos casos se deja de lado al usuario final, el estudiante, quien debe ser el protagonista del proceso de enseñanza-aprendizaje. En este trabajo se analiza el impacto del e-learning en los estudiantes mediante un estudio longitudinal que abarca los cursos 1999-2000 y 2005-2006. Se han analizado aspectos como la relación de los alumnos con las nuevas tecnologías, su satisfacción y su visión futura del e-learning. Los resultados indican que en 6 años ha aumentado la formación en nuevas tecnologías de los alumnos. Además, se puede concluir que el e-learning puede ser considerado como una alternativa válida a las clases presenciales.

Palabras clave: e-learning, nuevas tecnologías, satisfacción, aprendizaje, alumno.

\begin{abstract}
E-learning offers considerable advantages to organizations and universities as well as to users. There is a bull market for e-learning. In addition there are a great many initiatives to boost e-learning. However, some problems that make implementation of e-learning implementation difficult have been also detected. Studies about these issues are numerous but in many cases the final user, the student, is forgotten when he/she should be the protagonist of the teaching-learning process. This paper analyses the impact of e-learning on students by means of a longitudinal study that includes the academic years 1999-2000 and 2005-2006. Several issues such as the relationship of students with new technology, their satisfaction with and their future view of e-learning were analysed. Results suggest that in 6 years student skills in new technologies are increased. The results suggest that e-learning may be considered as a well-founded alternative to traditional classes
\end{abstract}

Keywords: e-learning, new technologies, satisfaction, learning, student. 


\section{DE LA EDUCACIÓN A DISTANCIA AL E-LEARNING}

La educación a distancia fue creciendo a lo largo del siglo XX como una vía alternativa de formación en la que no se exigiesen las rigideces espacio-temporales propias de la docencia convencional (García Aretio, 2001) dirigida a aquellas personas que, bien por su situación geográfica (alumnos en zonas rurales), sus condiciones de trabajo (personas con poco tiempo para atender una enseñanza reglada), sus condiciones físicas (personas con minusvalías) o bien por decisión personal, elegían una formación más acorde a sus necesidades (Marcelo et al., 2002).

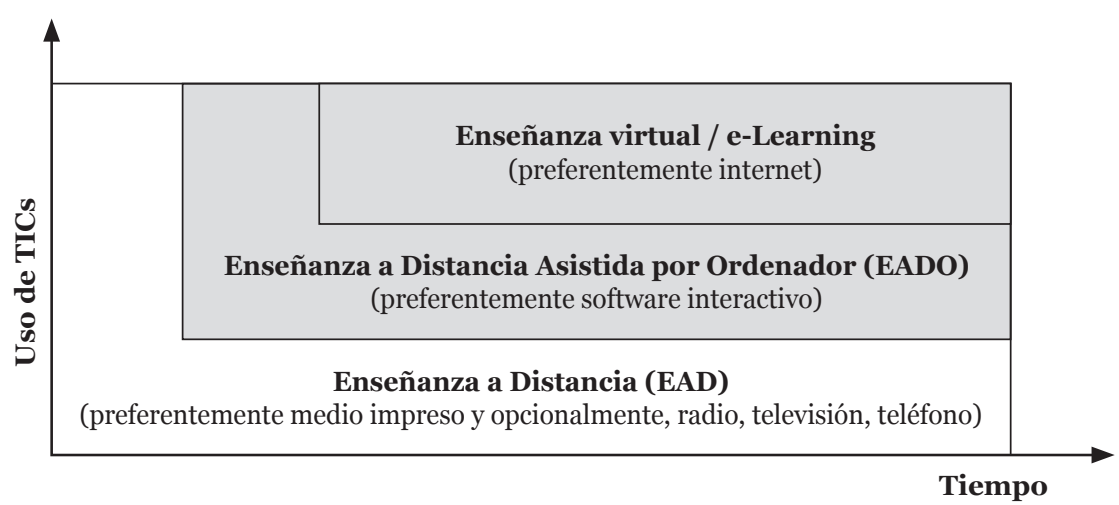

Figura 1. Etapas de la Educación a Distancia. Fuente: Elaboración Propia a partir de Ruipérez (2003)

A lo largo del tiempo se han realizado numerosos intentos por solucionar los problemas de la educación con las tecnologías de la comunicación. Estos intentos han marcado las grandes etapas de la Educación a Distancia las cuales se describen a continuación:

- Trascripción de las lecciones presenciales mediante la escritura a través de alfabetos, papiros y papel. La difusión de estos textos, que permitían a aprendices lejanos acceder a los conocimientos de historiadores, filósofos o dramaturgos, constituye uno de los primeros pasos en el desarrollo de los servicios postales.

- La invención de la imprenta en el siglo XV, permitió la disponibilidad de textos a una mayor audiencia. Sin embargo, no es hasta finales del siglo XIX, con la industrializaron de las sociedades, el desarrollo de redes eficientes de transporte, y la imprenta de alta velocidad, cuando se produce el verdadero desarrollo de la educación por correspondencia. 
- Radio, cine y televisión. Durante los años cuarenta y cincuenta se introdujeron el cine y la radio, y en los sesenta la televisión educativa.

- Medios de almacenamiento. El texto escrito empieza a estar apoyado por nuevos recursos audiovisuales (audiocasetes, videocasetes, diapositivas, etc.). Esta oferta se completó en los años ochenta con el uso del teléfono como medio para la realización de tutorías.

- Ordenadores personales. En los años 8o la educación se apoya en la proliferación de ordenadores personales y en programas de Enseñanza a Distancia Asistida por Ordenador.

- Internet. A finales de los noventa aparece la última de las innovaciones tecnológicas: la incorporación de Internet a la educación, dando lugar al eLearning. La figura 1 sintetiza las etapas citadas.

Se puede definir el e-learning como "una enseñanza apoyada en las tecnologías de la información y la comunicación donde no es necesario el encuentro físico entre profesores y alumnos y cuyo objetivo es posibilitar un aprendizaje flexible (a cualquier hora y cualquier lugar), interactivo (con comunicaciones síncronas y asíncronas) y centrado en el alumno" (Martínez-Caro, 2005).

El e-learning presenta numerosas ventajas, las cuales tienen unos claros beneficiarios: Las empresas, las universidades, los usuarios finales y, en definitiva, la sociedad. La Tabla 1 resume esas ventajas.

\begin{tabular}{|c|c|c|}
\hline Ventajas para las Empresas & Ventajas para las Universidades & Ventajas para los Usuarios \\
\hline $\begin{array}{l}\text { - Reduce un } 40-60 \% \text { de coste } \\
\text { respecto a la formación } \\
\text { tradicional }{ }^{1} \text {. } \\
\text { - Descentraliza la estructura } \\
\text { empresarial. Permite impartir } \\
\text { formación idéntica a todos los } \\
\text { empleados de un determinado } \\
\text { nivel, aunque trabajen en diferentes } \\
\text { localizaciones geográficas de la } \\
\text { misma empresa. } \\
\text { - Acceso a la formación de un mayor } \\
\text { número de trabajadores (masa } \\
\text { crítica). }\end{array}$ & $\begin{array}{l}\text { - Permite a la universidad ofertar } \\
\text { formación a las empresas sin } \\
\text { los añadidos que suponen los } \\
\text { desplazamientos, alojamientos y } \\
\text { dietas de sus trabajadores. } \\
\text { - Permite a la universidad ampliar } \\
\text { su oferta de formación a aquellas } \\
\text { personas o trabajadores que no } \\
\text { pueden acceder a sus cursos } \\
\text { presenciales. }\end{array}$ & $\begin{array}{l}\text { - Acceso en cualquier momento y en } \\
\text { cualquier lugar. Disponibilidad del } \\
\text { contenido } 24 \times 7 \text { ( } 24 \text { horas del día, } 7 \\
\text { días a la semana). } \\
\text { - Acceso a una amplia oferta formativa } \\
\text { al superar las barreras geográficas. } \\
\text { - Agilidad en la comunicación. } \\
\text { - Alumno como sujeto activo y } \\
\text { protagonista del proceso formativo. }\end{array}$ \\
\hline
\end{tabular}




\begin{tabular}{|l|c|c|}
\hline Ventajas para las Empresas & Ventajas para las Universidades & Ventajas para los Usuarios \\
\hline - Fácil acceso a la formación y & - Aumenta la efectividad de los & - Personalización del aprendizaje \\
actualización del personal de & presupuestos destinados a la & (contenidos, ritmos de aprendizaje, \\
la empresa, haciéndola más & educación: en muchos países los & tutorías personalizadas...). \\
competitiva y eficiente. & presupuestos de educación están & - Mayorinteracciónentreparticipantes \\
- Crea hábitos de uso de nuevas & congelados aunque la demanda & y profesores. Posibilidad de \\
tecnologías, que son aplicables & aumenta. & comunicación síncrona. \\
posteriormente en el trabajo diario. & - Responsabilidad del sistema & - Desarrollo de la formación en \\
- Genera una cultura de Internet, & educativo: los gobiernos no sólo & entornos colaborativos y dinámicos. \\
que trasforma la comunicación & esperan que las instituciones & \\
y relaciones internas y externas, & educativas mejoren su relación & \\
favoreciendocambios organizativos & coste-eficacia, sino que también & \\
y metodológicos. & esperan que éstas justifiquen el & \\
& uso que hacen del dinero público. & \\
\hline
\end{tabular}

Tabla 1. Ventajas del e-Learning para las Empresas, Universidades y Usuarios. Fuente: Elaboración Propia a partir de Bartolomé y Underwood (1998) y Formateca (2003)

Sin embargo, no todo son ventajas. Según Azcorra et al. (2001) y Formateca (2003) el e-learning también presenta ciertos inconvenientes, generalmente derivados de la corta edad de la misma, de su incorrecta concepción y de la falta de infraestructuras. En efecto, el uso de Internet como medio fundamental de transmisión de información tiene los problemas técnicos que la red aún presenta en la actualidad: escasez de ancho de banda en las redes, coste de conexión, etc... Aunque afortunadamente la banda ancha ha llegado a la mayoría de las empresas, no sucede lo mismo en los hogares ${ }^{2}$ lo que condiciona el desarrollo de contenidos y el uso de determinadas herramientas ya que implementar todos los recursos posibles hoy en día al alcance de la tecnología supone, en muchos casos para el alumno, un alto tiempo de espera, lo cual desalienta en el proceso formativo, pudiendo provocar abandonos.

Además, el e-learning continúa teniendo el problema de una cierta falta de confianza tanto por parte de las empresas como por parte del colectivo de profesores. Internet, las empresas y la filosofía "punto com" siguen considerándose como un medio no maduro. En general, se muestra una reticencia a pasar de la formación tradicional a un nuevo modelo de formación. Además, se tiende a pensar que la enseñanza virtual se limita a imitar las clases presenciales utilizando los medios de los que se dispone. Sin embargo, los diferentes actores involucrados (alumnos y profesores) deberían afrontar la enseñanza virtual no como un modo de imitar la enseñanza tradicional, sino como una nueva forma de aprendizaje, aprovechando las nuevas capacidades que ofrecen las redes de información y cambiando la forma de enseñar actual, tomando una parte más activa el alumno en su proceso de formación y no limitándose a actuar como mero receptor de información. 
Por otra parte, existe la creencia de que el e-learning es totalmente individualista y que presenta una falta de contacto humano entre alumnos y profesores. Sin embargo, el contacto utilizando las nuevas tecnologías puede ser más productivo y humano que el presencial. Ni un curso a distancia deja toda la responsabilidad en manos del alumno, ni un curso presencial está basado únicamente en el encuentro profesor-alumno.

Una vez descritas las principales características del e-learning, se evidencian las diferencias con respecto a la enseñanza presencial, poniéndose de manifiesto que éste no sólo aporta soluciones o alternativas para aquellas personas que se encuentran con dificultad para acceder a la enseñanza presencial sino que también ofrece elementos metodológicos y pedagógicos que le conceden valor por sí misma. En la Tabla 2 se resumen dichas diferencias.

\begin{tabular}{|c|c|c|}
\hline & Formación Tradicional & e-Learning \\
\hline Usuario & Sujeto pasivo & Sujeto activo \\
\hline Control del aprendizaje & Por parte del profesor & Por parte del alumno \\
\hline Espacio & Concentración geográfica & $\begin{array}{l}\text { Dispersión geográfica. El usuario puede estar } \\
\text { en cualquier lugar }\end{array}$ \\
\hline Tiempo & $\begin{array}{l}\text { Formación en el centro educativo } \\
\text { en un horario determinado }\end{array}$ & Acceso cuando quiere, según sus posibilidades \\
\hline Costes & De desplazamiento & De inversión en tecnología \\
\hline Requerimientos previos & No hay & Conocer básicamente la tecnología utilizada \\
\hline Enfoque & Colectivo & $\begin{array}{l}\text { Usuario individual (proceso personalizado, } \\
\text { tutorías personalizadas) y usuarios colectivos } \\
\text { (trabajo en grupo) }\end{array}$ \\
\hline
\end{tabular}

Tabla 2. La Formación Tradicional frente al e-Learning. Fuente: Adaptado de Formateca (2003)

\section{SITUACIÓN ACTUAL DEL E-LEARNING}

La aparición del e-learning ha sido desigual a nivel mundial, pudiendo establecerse tres grupos (Azcorra et al., 2001):

- Madurez. Formado por aquellos países en los que el e-learning se encuentra en un grado de madurez aceptable, en los que además de existir una amplia oferta, se empieza a observar una gran participación del e-learning en el sector educativo (más de un 10\%). Entre estos países cabe destacar a EEUU, Australia, Reino 
Unido, Irlanda y Canadá. Por lo general estos países suelen ser los que tienen más recursos económicos así como tecnológicos. Poseen una alta implantación de las nuevas tecnologías, y ello sin duda ha contribuido a que sean los que estén a la cabeza del e-learning mundial. Existen ausencias significativas como Francia y Alemania en los que el desarrollo de la enseñanza virtual no está a la altura de los países mencionados antes. Uno de los motivos podría ser el idioma, que restringe las experiencias prácticamente a las fronteras nacionales.

- Desarrollo. Este grupo incluye los países en los que el e-learning está empezando o bien no se ha alcanzado un desarrollo total. En él se encuentra la mayoría de los países de la UE (excepto los anteriormente mencionados) y los países suramericanos. Se caracterizan bien por no poseer una lengua de extenso uso mundial (Alemania, Francia, o Italia) o bien porque el desarrollo y, sobre todo, la implantación de las nuevas tecnologías en dichos países, ha sido ligeramente tardío (como en España).

- Inicio. El tercer grupo lo forman países en los que el e-learning apenas existe debido al escaso poder tecnológico de los mismos (por ejemplo, la mayoría de los países africanos y asiáticos) o bien porque se han incorporado tarde al desarrollo de las nuevas tecnologías (por ejemplo, los países del este incluyendo Rusia).

Actualmente el e-learning representa el 11\% de la formación en España. El año 2006 fue un año de fuerte crecimiento del e-learning, paralelo al del aumento del presupuesto de formación en las empresas, con un incremento por encima del 30\%, alcanzando los 165 millones de euros (Élogos, 2006). Aunque las previsiones sobre el crecimiento del e-learning varían, a nivel general indican que las organizaciones seguirán aumentando el uso del e-learning (O’Leonard, 2007).

A pesar de este crecimiento, hay que tener en cuenta que el cambio social que ha provocado el e-learning todavía está en progreso y se requiere el tiempo necesario para adaptar el sistema de aprendizaje a este nuevo entorno formativo. No obstante, está claro que todas las clases de compañías e instituciones, aunque no estén directamente relacionadas con el e-learning, deben revisar su estrategia y adaptarse a los retos derivados de este nuevo modo de tratar la formación (ICT, 2003). En ese sentido, son numerosas las iniciativas para el impulso del e-learning.

Así, la UNESCO puso de manifiesto la necesidad de extender las nuevas tecnologías como herramienta de democratización de la educación. En la Conferencia 
Mundial de Educación Superior (CMES), se puso de manifiesto la necesidad de implementar las nuevas tecnologías en las universidades. Este fue uno de los temas claves que se discutieron y que están constatados en la Declaración de la $\mathrm{CMES}^{3}$, en Octubre de 1998, en París. A su vez, la UNESCO 4 recomienda el e-learning como una herramienta que permite poner el conocimiento al alcance de todo el mundo, y en este sentido, deben vehicularse las acciones que desarrollan los distintos gobiernos y organismos competentes.

En 2003, la Universidad de las Naciones Unidas (UNU), puso en marcha la iniciativa Universidad Virtual Global ${ }^{5}$ con el objetivo de virtualizar muchos de sus programas educativos. Se trata de una universidad virtual enmarcada dentro de los planes de desarrollo sostenible de la Naciones Unidas, con un marcado enfoque hacia los países mas desfavorecidos. Está constituida por una red internacional de universidades e instituciones que actúan como sucursales de la UNU y por una administración central con sede en Noruega.

En el año 2000, la Comisión Europea emprendió la iniciativa "eLearning - Concebir la educación del futuro", donde señala el potencial del e-learning para ayudar a la Unión a dar respuesta a los retos de la sociedad del conocimiento, mejorar la calidad del aprendizaje, facilitar el acceso a los recursos de aprendizaje, satisfacer necesidades especiales, y permitir un aprendizaje y una formación más eficaces en el lugar de trabajo, en particular en las pequeñas y medianas empresas. En diciembre de 2003 se adoptó el programa "E-Learning" plurianual (2004-2006) para la integración efectiva de las TIC en los sistemas de educación y formación de Europa ${ }^{6}$.

El gobierno de los Estados Unidos también ha desarrollado medidas para fomentar la implantación de una educación virtual. Las políticas de e-learning se desarrollan a través de la política tecnológica y de la política educativa. En 2004 se lanzó el Plan Nacional de Educación Tecnológica ${ }^{7}$ que tenía como uno de sus objetivos la promoción del e-learning y de las escuelas virtuales.

Existen en el mundo diversas asociaciones que trabajan para la promoción del elearning. Son destacables la $\mathrm{AACE}^{8}$ (Association for the Advancement of Computing in Education) o la EADL $^{9}$ (European Association for Distance Learning).

En España destacan tres asociaciones dedicadas al e-learning: AEDISI ${ }^{10}$ (Agencia Española para el Desarrollo e Investigación de la Sociedad de la Información), AEFOL $^{11}$ (Asociación Española para la Formación On-line) y APEL ${ }^{12}$ (Asociación de Proveedores de e-learning). 
Por otra parte, la Ley Orgánica de Universidades (LOU), aprobada en diciembre de 2001, da un respaldo al e-learning animando a las universidades españolas a la creación de sus propios "centros o estructuras que organicen enseñanzas en modalidad no presencial" (Art. 7.1). Asimismo, contempla la "creación de un Centro Superior para la Enseñanza Virtual específicamente dedicado a esta modalidad de enseñanza en los distintos ciclos de los estudios universitarios" (disposición adicional 2.3) que debería convertirse en centro de formación e investigación de referencia de las universidades.

\section{ESTUDIO EMPÍRICO}

\section{Objetivos}

Como se ha expuesto anteriormente, el e-learning se presenta como una alternativa a la enseñanza tradicional con grandes posibilidades. Se han descrito las ventajas que ofrece tanto a las organizaciones y universidades como a los usuarios, se ha puesto de manifiesto la tendencia alcista del mercado del e-learning y se han citado las numerosas iniciativas existentes para el impulso del e-learning. Sin embargo, también se han detectado algunos problemas e inconvenientes a los que se enfrenta el e-learning y que dificulta su implantación. En este sentido, se han realizado grandes avances a nivel tecnológico (como el desarrollo de plataformas para la gestión del aprendizaje o LMS así como el de estándares), a nivel de servicios (por ejemplo, la proliferación de proveedores de servicios de aplicaciones o $\mathrm{ASP}^{13}$ ) y a nivel de contenidos (por ejemplo, el desarrollo de contenidos reutilizables). En relación con estos avances, son numerosos los estudios que tratan sobre esos aspectos pero en muchos casos se deja de lado al usuario final, el estudiante, quien debe ser el protagonista del proceso de enseñanza-aprendizaje. En este trabajo se ha pretendido abordar esa perspectiva analizando el impacto de la formación e-learning en los estudiantes. Para ello se han analizado aspectos relativos a la relación de los alumnos con las nuevas tecnologías, su satisfacción y su visión futura del e-learning.

\section{Muestra}

Se ha realizado un estudio longitudinal donde participaron dos grupos de alumnos matriculados en la asignatura troncal "Organización Empresarial y Administración de la Producción" impartida en $4^{\circ}$ curso de la titulación Ingeniero Industrial, en los cursos académicos 1999-2000 y 2005-2006 respectivamente. La muestra, la 
tasa de respuesta así como algunos datos sociodemográficos de los participantes se muestran en la Tabla 3.

\begin{tabular}{cccccc}
\hline Curso & Muestra & $\begin{array}{c}\text { Tasa de } \\
\text { respuesta }\end{array}$ & Edad media & $\begin{array}{c}\text { Género } \\
\text { (\% hombres) }\end{array}$ & Trabajadores \\
\hline $1999-2000$ & 27 & $90 \%$ & 23,6 & $86,6 \%$ & $26,6 \%$ \\
$2005-2006$ & 40 & $91 \%$ & 22,6 & $85 \%$ & $32,5 \%$ \\
\hline
\end{tabular}

Tabla 3. Muestra del estudio y sus características

\section{Descripción de los cursos e-learning analizados}

Para impartir la asignatura en modo e-learning se utilizóla plataforma tecnológica Lotus LearningSpace ${ }^{\circledR} 3.5$ durante el curso 1999-2000 y la plataforma tecnológica WebCT ${ }^{\circledR} 4.0$ en el curso 2005-2006. En ambas ocasiones se proporcionó a los alumnos información general sobre la asignatura y sobre el profesorado, así como los materiales educativos en diversos formatos (texto, gráficos, texto interactivo, video, etc.). La evaluación se realizó de manera continua mediante la resolución de ejercicios y la realizaron de exámenes tipo test. En ambos cursos se emplearon herramientas de comunicación asíncrona, como el correo electrónico y los foros de debate. Las tutorías se realizaron a través del correo electrónico, el foro de debates, el teléfono o el chat. Al inicio de la asignatura se mantuvo una sesión presencial con los alumnos en la que se les informó sobre el modo de funcionamiento de la misma y se les explicó cómo utilizar la plataforma tecnológica empleada para impartirla. Asimismo se realizó un encuentro presencial al final de la misma en la cuál se efectuó la recogida de datos mediante un cuestionario semiestructurado de 43 items, en el que se incluyeron 4 preguntas abiertas, 6 dicotómicas y 33 categorizadas. De las preguntas categorizadas, 31 utilizaban una escala de respuesta tipo Likert de 5 puntos.

\section{Resultados}

Los conocimientos informáticos de los alumnos de ambos grupos, antes de participar en la experiencia e-learning, tienen una media similar que indica unos conocimientos medios (Tabla 4). 


\begin{tabular}{|l|c|c|c|c|}
\hline \multirow{2}{*}{ Relación alumnos con Nuevas Tecnologías } & \multicolumn{2}{|c|}{ Curso 1999-2000 } & \multicolumn{2}{c|}{ Curso 2005-2006 } \\
\cline { 2 - 5 } & Media & Desv. Típica & Media & Desv. Típica \\
\hline Conocimientos informáticos previos & 3,3 & 0,8 & 3,5 & 0,8 \\
Conocimientos informáticos posteriores & 3,8 & 0,6 & 4,0 & 0,5 \\
Experiencia en Internet previa & 2,3 & 0,7 & 3,6 & 0,8 \\
Experiencia en Internet posterior & 3,9 & 0,8 & 4,1 & 0,6 \\
\hline
\end{tabular}

* Las variables representadas se han medido mediante una escala de respuesta que toma valores comprendidos entre 1 y 5 , siendo 3 el valor medio de dicha escala.

Tabla 4. Relación alumnos con Nuevas Tecnologías (I): Conocimientos informáticos y experiencia en Internet

Sin embargo, la distribución de frecuencias muestra que mientras el 51,85\% de los alumnos del curso 1999-2000 creían tener unos conocimientos medios y el 48,15\% unos conocimientos básicos, en el curso 2005-2006, el 55\% los alumnos consideraban tener unos conocimientos informáticos elevados y sólo el 12,5\% unos conocimientos básicos (Figura 2).

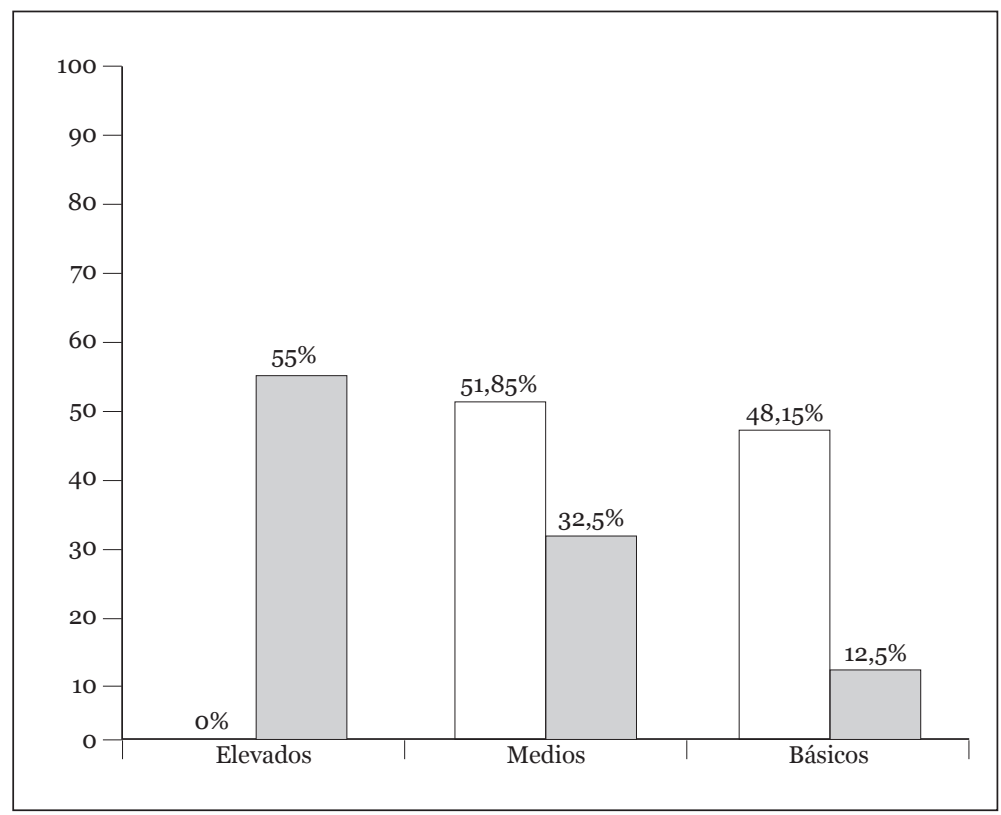

Figura 2. Conocimientos informáticos previos de los alumnos. Comparativa de la distribución de frecuencias en los dos cursos analizados 
En ambos casos, dichos conocimientos aumentaron tras realizar el curso en modalidad e-learning. De manera similar, en el caso de la experiencia en Internet antes de participar en la experiencia e-learning era más baja en el curso 1999-2000 que en el curso 2005-2006, aumentado en ambos casos tras el curso e-learning.

Con respecto a la disponibilidad de conexión a Internet en casa, se observa que ha aumentado notablemente en los 6 años transcurridos entre una experiencia y otra (Tabla 5), alcanzado el 80\% en el curso 2005-2006. Además, estos porcentajes aumentaron tras la experiencia e-learning en ambos cursos. En relación al lugar desde el que se conectaron habitualmente a Internet los alumnos durante el curso, lo hicieron fundamentalmente desde casa, ocupando la Universidad el segundo lugar.

\begin{tabular}{|l|c|c|}
\hline Relación alumnos con Nuevas Tecnologías & Curso 1999-2000 & Curso 2005-2006 \\
\hline Conexión en casa previa & $63 \%$ & $80 \%$ \\
Conexión en casa posterior & $74 \%$ & $87,5 \%$ \\
\hline Lugar habitual de conexión & $59,2 \%$ & $77,5 \%$ \\
\hline Casa & $25,9 \%$ & $17,5 \%$ \\
Universidad & $11 \%$ & $2,5 \%$ \\
Trabajo & $3,7 \%$ & $2,5 \%$ \\
Otros & $100 \%$ & $72,5 \%$ \\
Primera experiencia e-learning & \\
\hline
\end{tabular}

Tabla 5. Relación con Nuevas Tecnologías (I): Hábitos de navegación y experiencias e-learning

Otro aspecto de importancia es el hecho de que mientras que en el curso 19992000 todos los alumnos participaban por primera vez en una asignatura impartida en la modalidad e-learning en el curso 2005-2006, el 27,5\% de los alumnos ya habían tenido esa experiencia.

Entre los motivos que llevaron a los alumnos a participar en la experiencia elearning, destacan en ambos cursos el evitar desplazamientos y el poder solucionar conflictos de horarios (Tabla 6). Por otra parte, tanto en el curso 1999-2000 como en el 2005-2006, los alumnos consideraron con puntuaciones altas que fue fácil adaptarse al sistema e-learning y que estaban satisfechos con el contacto que habían tenido con el profesor así como con los demás alumnos (aunque en este último caso con puntuaciones ligeramente inferiores). Como ventajas del e-learning, ambos grupos de alumnos señalaron la libertad de horarios, el poder aprender a su propio ritmo y el poder compaginar el estudio con otras actividades como el trabajo. Como 
inconvenientes señalaron la necesidad de una mayor disciplina y los problemas técnicos relacionados con Internet y el software empleado para impartir la asignatura, aunque a estos inconvenientes le otorgaron menor importancia.

\begin{tabular}{|l|c|c|c|c|}
\hline \multirow{2}{*}{ Resultados respecto a la modalidad e-learning } & \multicolumn{2}{|c|}{ Curso 1999-2000 } & \multicolumn{2}{c|}{ Curso 2005-2006 } \\
\cline { 2 - 5 } & Media & Desv. Típica & Media & Desv. Típica \\
\hline Motivos & 3,9 & 1,2 & 3,3 & 1,5 \\
\hline Evitar desplazamientos & 3,5 & 1,0 & 3,9 & 1,1 \\
Conflictos de horarios & 1,7 & 0,9 & 1,5 & 0,9 \\
Responsabilidades familiares/laborales & 4,0 & 0,5 & 2,6 & 1,8 \\
Otros & 3,9 & 0,9 & 4,0 & 0,8 \\
Facilidad de adaptación & 3,9 & 0,9 & 4,0 & 0,7 \\
Satisfacción contacto profesor & 3,8 & 0,9 & 3,5 & 0,8 \\
Satisfacción contacto alumnos & 4,4 & 0,8 & 4,4 & 0,6 \\
\hline Ventajas & 4,4 & 0,9 & 4,3 & 0,8 \\
\hline Libertad de horarios & 4,4 & 0,8 & 4,4 & 0,7 \\
Aprender a mi propio ritmo & \multicolumn{5}{|}{} \\
Compaginar estudio-otras actividades & 3,0 & 0,9 & 3,7 & 0,8 \\
\hline Inconvenientes & 3,8 & 1,0 & 2,7 & 0,9 \\
\hline Necesito mayor disciplina & \multicolumn{5}{|l|}{} \\
\hline Problemas técnicos
\end{tabular}

* Las variables representadas se han medido mediante una escala de respuesta que toma valores comprendidos entre 1 y 5 , siendo 3 el valor medio de dicha escala.

Tabla 6. Resultados respecto a la modalidad e-learning: motivación, satisfacción con la interacción entre participantes, ventajas e inconvenientes

Como resultados globales cabe señalar que en ambos casos los alumnos presentaron un alto grado de satisfacción con el e-learning (medias de 4,21 y 4,11, respectivamente) (Tabla 7). Otro aspecto de gran relevancia, es el aprendizaje de los alumnos. Para medir esta variable se optó por utilizar una medida subjetiva: el aprendizaje percibido por los alumnos. Aunque tradicionalmente los investigadores han optado por emplear las puntuaciones obtenidas por los alumnos en determinadas pruebas de evaluación como un indicador del aprendizaje adquirido por los mismos, usar esas notas para determinar el aprendizaje no proporciona siempre los mejores resultados. Dichas notas están sujetas a una serie de influencias como la participación en clase, la asistencia, la habilidad para escribir, los trabajos entregados fuera de plazo, etc.

McCroskey y Richmond (1992) rechazan la opción de emplear las notas de los alumnos porque no reflejan el grado en el que éstos pueden aprender. Por ejemplo, las puntuaciones pueden estar afectadas por lo que los participantes saben al iniciar 
el curso y no muestran lo que han aprendido durante el mismo. Por otro lado, puede que las notas no sean una medida válida del aprendizaje, en particular en el caso de pruebas no objetivas que son corregidas por profesores diferentes o incluso por el mismo profesor pero en momentos distintos, quienes probablemente no asignen las notas de manera consistente (Rovai, 2002).

Ante la ausencia de una medida del aprendizaje sólida y objetiva, Richmond et al. (1987) proponen una medida subjetiva: el aprendizaje percibido por los alumnos. Aunque es razonable esperar que los estudiantes sean capaces de opinar sobre su grado de motivación o cuánto les gusta un profesor o una clase, no se da por hecho que los alumnos puedan determinar cuánto han aprendido. Sin embargo, los estudiantes de ciclos superiores, son adultos con bastante experiencia en el mundo educativo (Chesebro y McCroskey, 2000). Richmond et al. (1987) creen que es razonable considerar que estos estudiantes pueden estimar con bastante exactitud cuánto han aprendido en las clases recibidas. De hecho, opinan que se puede considerar que esta estimación es al menos tan buena como las notas subjetivas proporcionadas por algunos profesores o que son resultado de pruebas que no están basadas en unos objetivos claros. En definitiva, ninguno de estos métodos es inherentemente superior a otros en términos de validez. Más bien, cada uno de ellos mide el aprendizaje de maneras diferentes.

En la presente investigación, se ha optado por medir el aprendizaje mediante una adaptación del instrumento propuesto por Richmond et al. (1987): los alumnos deben responder a la pregunta "Indique cuánto ha aprendido en esta asignatura, teniendo en cuenta que una puntuación igual a 1 significa que ha aprendido menos que en otras asignaturas presenciales que haya realizado y una puntuación igual a 5 que ha aprendido más".

\begin{tabular}{|c|c|c|c|c|c|c|c|c|}
\hline Resultados globales & \multicolumn{4}{|c|}{ Curso 1999-2000 } & \multicolumn{4}{|c|}{ Curso 2005-2006 } \\
\hline \multirow{2}{*}{$\begin{array}{l}\text { Satisfacción general con } \\
\text { e-learning }\end{array}$} & \multicolumn{2}{|c|}{ Media } & \multicolumn{2}{|c|}{ Desv. Típica } & \multicolumn{2}{|c|}{ Media } & \multicolumn{2}{|c|}{ Desv. Típica } \\
\hline & \multicolumn{2}{|c|}{4,2} & \multicolumn{2}{|c|}{0,6} & \multicolumn{2}{|c|}{4,1} & \multicolumn{2}{|c|}{0,6} \\
\hline \multirow{2}{*}{$\begin{array}{l}\text { Aprendizaje respecto a otras } \\
\text { clases }\end{array}$} & Menos & & ual & Más & Menos & & ual & Más \\
\hline & $11,1 \%$ & & $2 \%$ & $29,6 \%$ & $2,6 \%$ & & $5 \%$ & $57,9 \%$ \\
\hline \multirow{2}{*}{$\begin{array}{l}\text { Posibilidad de expansión del } \\
\text { e-learning }\end{array}$} & No & Sí & $\begin{array}{c}\mathrm{S} / \\
\text { profesor }\end{array}$ & $\begin{array}{c}\mathrm{S} / \\
\text { materia }\end{array}$ & No & Sí & $\begin{array}{c}\text { S/ } \\
\text { profesor }\end{array}$ & $\begin{array}{c}\mathrm{S} / \\
\text { materia }\end{array}$ \\
\hline & $0 \%$ & $0 \%$ & $26 \%$ & $74 \%$ & $0 \%$ & $15 \%$ & $7,5 \%$ & $72,5 \%$ \\
\hline
\end{tabular}

* La variable satisfacción se ha medido mediante una escala de respuesta que toma valores comprendidos entre 1 y 5 , siendo 3 el valor medio de dicha escala.

Tabla 7. Resultados globales del curso 
En ambos casos, un gran porcentaje de los alumnos consideró que había aprendido igual o más que en la enseñanza presencial ( $88,87 \%$ y 97,36\% respectivamente). Finalmente, ante la posibilidad de la expansión del e-learning como una alternativa a la formación tradicional, de manera general, los alumnos creen que es posible pero dependiendo de la materia, considerando que serían preferibles las materias con menor contenido práctico o bien, optar por una fórmula blended-learning, de manera que la parte teórica se impartiera en la modalidad e-learning y la parte práctica de forma presencial.

\section{CONCLUSIONES}

En este trabajo se ha presentado el e-learning como una alternativa a la enseñanza tradicional con grandes posibilidades. Asimismo se ha pretendido analizar el impacto de la formación e-learning en los usuarios finales, los estudiantes, quienes son los protagonistas del proceso de enseñanza-aprendizaje. Para ello se han analizado las opiniones de 67 alumnos que participaron en dos experiencias e-learning: un primer grupo en el curso 1999-2000 y un segundo grupo en el curso 2005-2006, con el objetivo de observar si había diferencias entre los mismos trascurridos 6 años entre una experiencia y otra.

Así, se observa que en ese período la relación de los alumnos con las nuevas tecnologías ha aumentado: los alumnos actuales poseen mayores conocimientos informáticos y una mayor experiencia en Internet que los alumnos que les precedieron 6 años antes. Además se han detectado diferencias con respecto al número de conexiones a Internet disponible en los hogares, habiendo aumentado asimismo en los últimos 6 años. Por otra parte, es interesante comprobar como el e-learning se ha ido expandiendo durante estos años ya que mientras que el 100\% de los alumnos del curso 1999-2000 participaban por primera vez en un curso e-learning, este porcentaje se había reducido al 72,5\% en el curso 2005-2006. Estos datos reflejan cómo el elearning ha ido introduciéndose e integrándose en la formación universitaria.

En ambos grupos se ha detectado un aumento en los conocimientos informáticos, el uso de Internet y el número de conexiones tras participar en la experiencia elearning. Teniendo en cuenta estos resultados se puede afirmar que el e-learning fomenta el uso de las nuevas tecnologías y crea una cultura de Internet preparando a los alumnos para formar parte de la sociedad del conocimiento y facilitando su acceso al mercado laboral en el que estas competencias están cobrando una gran importancia. 
Centrándonos en aspectos relacionados con el e-learning, cabe señalar que las motivaciones principales por las que los alumnos de ambos grupos participaron en la experiencia e-learning fueron principalmente el hecho de evitar desplazamientos y el poder solucionar conflictos de horarios, es decir, la flexibilidad de la formación e-learning. En definitiva, la ruptura de la rigidez espacio-temporal propia de la enseñanza presencial, es lo que más se valora. En general, consideraron que había sido fácil adaptarse al sistema e-learning, independientemente de sus conocimientos informáticos o experiencia previa en Internet. Esto supone que las carencias en dichos conocimientos o competencias no son un impedimento a la hora de optar por la formación e-learning. Por otra pare, los alumnos señalaron que estaban satisfechos con el contacto que han tenido con el profesor así como con los demás alumnos, desmitificando la creencia de que el e-learning es totalmente individualista y que presenta una falta de contacto humano entre alumnos y profesores. Como ventajas del e-learning, los alumnos destacaron la libertad de horarios, el poder aprender a su propio ritmo y el poder compaginar el estudio con otras actividades como el trabajo, incidiendo nuevamente en la flexibilidad del e-learning. Como inconvenientes señalaron, aunque con menor importancia, la necesidad de una mayor disciplina y los problemas técnicos relacionados con Internet y el software empleado para impartir la asignatura. El primer inconveniente puede estar relacionado con la madurez y la motivación del alumno, y sería conveniente profundizar en su análisis en futuras investigaciones. Los problemas relacionados con problemas técnicos, deberían desaparecer conforme mejore la calidad de las conexiones a Internet y vaya madurando el software empleado. No obstante, si se comparan los datos de ambos grupos, se detecta que esa tendencia a disminuir los problemas técnicos ya existe.

Los resultados globales confirman que el e-learning es una alternativa válida a la enseñanza presencial, ya que los alumnos muestran un alto grado tanto de satisfacción como de aprendizaje siendo incluso este último mayor que el que los alumnos perciben en las clases presenciales. Finalmente, los alumnos opinaron respecto al futuro del e-learning que seguiría creciendo pero aplicándolo a materias de carácter principalmente teórico o bien, bajo la fórmula de blended-learning. Efectivamente, la opción blended-learning se ha ido expandiendo, pero también hay que tener en cuenta el avance en desarrollo de contenidos e-learning, que cada vez son de mayor calidad. Un ejemplo de ello es la inclusión de simuladores y laboratorios virtuales, que permiten ofrecer en la modalidad e-learning materias técnicas o experimentales.

Finalmente, a la hora de generalizar los resultados de este trabajo hay que considerar el hecho de que todos los trabajadores de la muestra tienen un perfil 
técnico (estudiantes de ingeniería). Asimismo, el profesorado de todos los programas ha sido el mismo, lo que puede tener dos lecturas distintas: en primer lugar puede aumentar la validez interna del estudio puesto que se elimina cualquier diferencia que habrían podido causar los diferentes estilos de enseñanza de los profesores en el caso de que el profesorado hubiera sido distinto en cada programa; por otra parte, este escenario no sería totalmente representativo de una situación real, pudiendo haber una menor validez externa que disminuye la capacidad de generalización de los resultados. En futuros estudios habría que analizar asignaturas que versen sobre otras materias y que estén impartidos por distinto profesorado.

\section{NOTAS}

1 Según Formateca (2003) el e-learning supone un ahorro de costes de un 40-60\% respecto a la enseñanza tradicional, tanto directos (costes propios de la formación) como indirectos (desplazamientos, comidas, horas de trabajo, etc.).

Según datos del INE (Instituto Nacional de Estadística), en España el porcentaje de empresas con conexión de banda ancha ha aumentado desde un $62,41 \%$ en 2002 a un 95,23\% en 2007. Por otra parte, en el caso de los hogares españoles ese porcentaje ha variado desde un 2,05\% en 2002 a un 39,2\% en 2007. http://www.unesco.org/education/wche/declaration.shtml http://www.unesco.org/education/portal/e learning/index.shtml http://www.gvu.unu.edu

6 Decisión $\mathrm{n}^{\mathrm{o}}$ 2318/2003/CE del Parlamento Europeo y del Consejo de 5 de diciembre de 2003 (http://eur-lex.europa.eu/LexUriServ/site/es/oj/2003/l 345/ l 34520031231es00090016.pdf) http://www.ed.gov/about/offices/list/os/technology/plan/2004/site/edlite-default. $\underline{\mathrm{html}}$

http://www.aace.org

http://www.eadl.org

http://www.aedisi.org

http://www.aefol.com

http://www.apel.es

Empresa que permite a sus clientes el uso de aplicaciones software a través de una conexión segura de Internet mediante un modelo de alquiler o cuota periódica. Esto implica que los servicios y aplicaciones software no están instalados en la oficina del cliente, sino en la empresa proveedora del servicio o en un data center contratado por ella (Selma, 2004). 


\section{REFERENCIAS BIBLIOGRÁFICAS}

AEFOL (2003). El Estado del e-Learning en España. Barcelona: AEFOL.

Azcorra, A.; Bernardos, C. J.; Gallego, O.; Soto, I. (2001). Informe sobre el Estado de la Teleeducación en España. Mundo Internet, Febrero 2001, 301380 [en línea] Disponible en: http:// www.it.uc3m.es/ azcorra/papers/ teleeducacion mundoineto1.pdf [consulta 2007, 14 de abril].

Bartolomé, A. R.; Underwood, J. D. M. (1998). TEEODE. Technology Enhanced Evaluation in Open and Distance Education. Barcelona: Universidad de Barcelona.

Chesebro, J. L.; McCroskey, J. C. (2000). The Relationship between Students Reports of Learning and their Actual Recall of Lecture Material: A Validity Test. Communication Education, 49, 3, 297-301.

Élogos (2006). El Estado del Arte de la Formación en España.

Formateca (2003). E-Learning. Visión y Tendencias. Albacete: Génesis XXI.

García Aretio, L. (2001). La Educación a Distancia. De la Teoría a la Práctica. Barcelona: Ariel Educación.

InstitutCatalàdeTecnología(2003).Situación y Prospectiva del E-Learning en España. E-learning for Training in Information and Communication Technologies Project. [en línea] Disponible en: http://www.telecentros.info/pdfs/ SITUACION PROSPECTIVA ESP.pdf [consulta 2006, 5 de diciembre].

Marcelo, C.; Puente, D.; Ballesteros, M. A.; Palazón, A. (2002). E-Learning - Teleformación. Diseño y Desarrollo de la Formación a través de Internet. Barcelona: Gestión 2000.
Martínez-Caro, E. (2005). La Mejora de la Calidad en la Educación mediante Entornos Virtuales de Aprendizaje. Tesis Doctoral, Universidad Politécnica de Cartagena.

McCroskey, J. C.; Richmond, V. P. (1992). Increasing Teacher Influence through Immediacy, en: Richmond, V. P.; McCroskey, J. C. (Eds.), Power in the Classroom: Communication, Control and Concern. Hillsdale, New York: Lawrence Erlbaum.

O'Leonard, K. (2007). The corporate learning factbook. California: Bersin \& Associates.

Richmond, V. P.; McCroskey, J. C.; Kearney, P.; Plax, T. G. (1987). Power in Classroom VII: Linking Behaviour Alteration Techniques to Cognitive Learning. Communication Education, 36, 1, 1-12.

Rovai, A. P. (2002). Sense of Community, Perceived Cognitive Learning, and Persistence in Asynchronous Learning Networks. Internet and Higher Education, 5, 319-332.

Ruipérez, G. (2003). Educación Virtual y eLearning. Madrid: Biblioteca AUNA Fundación.

Selma, E. (2004). El Modelo ASP. Actualidad TIC, 5. [en línea] Disponible en: http://www.iti.upv. es/actualidadtic/2004/10/2004-10portada.pdf [consulta 2007, 9 de mayo]. 


\section{PERFIL ACADÉMICO Y PROFESIONAL DE LA AUTORA}

Eva Martínez Caro. Doctora por la Universidad Politécnica de Cartagena. Es profesora del Departamento de Economía de la Empresa de dicha universidad, impartiendo docencia en la Escuela Técnica Superior de Ingenieros Industriales. Sus líneas de investigación se centran en la aplicación de las nuevas tecnologías en la educación, la gestión del conocimiento y la calidad. Ha sido responsable de la implantación y desarrollo del Aula Virtual de la Universidad Politécnica de Cartagena desde 2001 hasta 2006.

E-mail: eva.martinez@upct.es

DIRECCIÓN DE LA AUTORA:

Campus Muralla del Mar

Universidad Politénica de Cartagena

$\mathrm{C} /$ Doctor Fleming, $\mathrm{s} / \mathrm{n}$

30203 Cartagena (Murcia)

España

Fecha de recepción del artículo: 12/12/07

Fecha de aceptación del artículo: 10/03/08 